
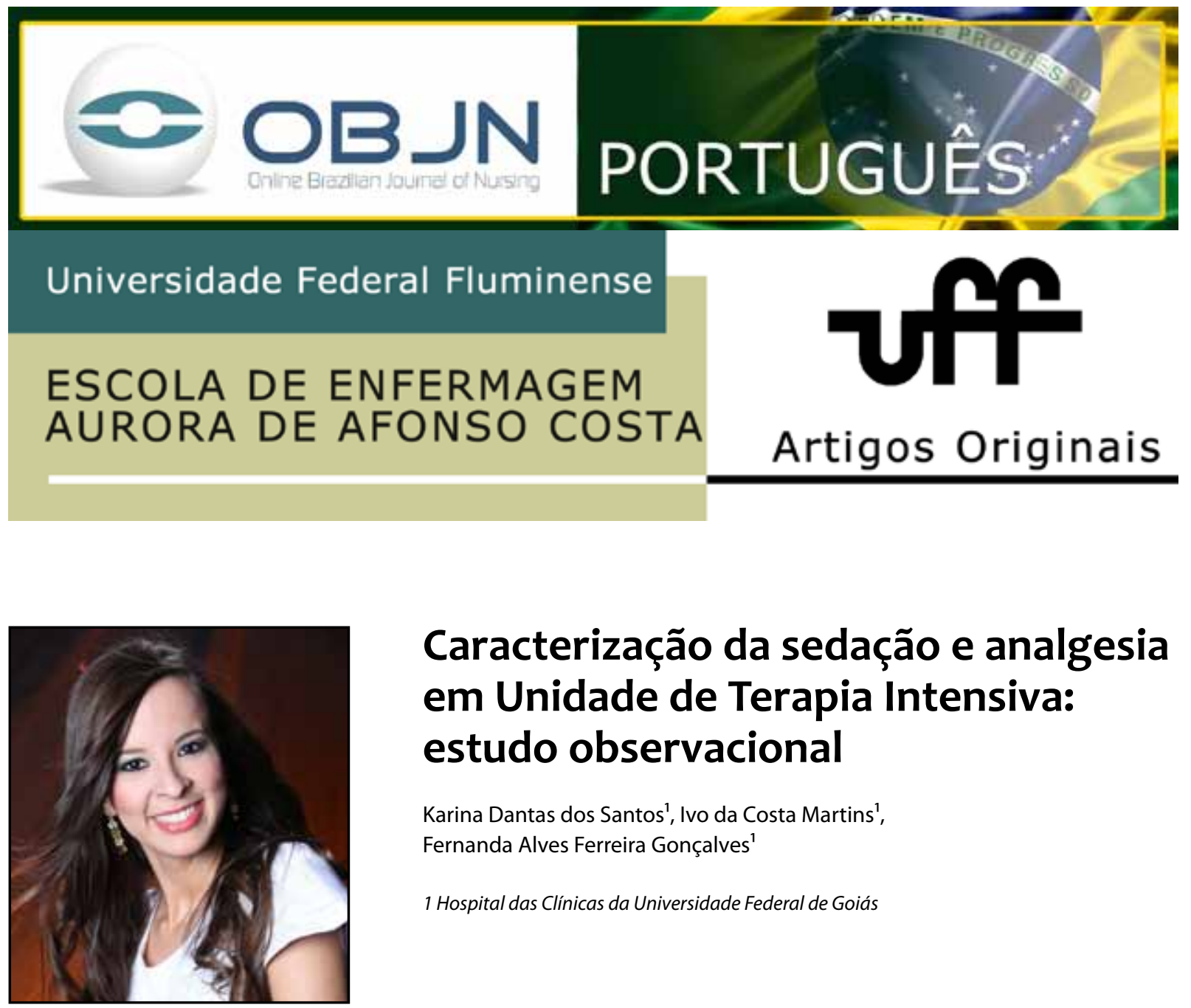

\title{
Caracterização da sedação e analgesia em Unidade de Terapia Intensiva: estudo observacional
}

\author{
Karina Dantas dos Santos ${ }^{1}$, Ivo da Costa Martins' ${ }^{1}$, \\ Fernanda Alves Ferreira Gonçalves ${ }^{1}$ \\ 1 Hospital das Clínicas da Universidade Federal de Goiás
}

\section{RESUMO}

Objetivo: avaliar o processo de sedação e analgesia em pacientes sob ventilação mecânica invasiva (VMI). Método: estudo transversal, observacional, realizado de setembro de 2014 a fevereiro de 2015 na UTI de uma instituição de ensino de Goiânia/GO. A amostra foi constituída de 30 pacientes maiores de 18 anos, intubados e sedados há mais de 24 horas (protocolo $n^{\circ}$. 763.827/2014). Resultados: foram utilizadasanalgesia e sedação contínua e intermitente, sendo o fentanil e o dormonid as drogas mais usadas. Discussão: o tratamento padrão entre pacientes críticos e submetidos à VMI foi a sedação contínua, administrada devido à ansiedade, para facilitar o cuidado da equipe de enfermagem. A principal indicação para sedação foi a manutenção de VMI. Conclusão: falta padronização das condutas no manejo da sedação. Recomenda-se a elaboração de protocolos com atuação da equipe multiprofissional.

Descritores: Hipnóticos e Sedativos; Terapia Intensiva; Analgesia; Sedação Profunda. 


\section{INTRODUÇÃO}

O internamento na Unidade de Terapia Intensiva (UTI) está associado geralmente a diversos procedimentos invasivos (ventilação mecânica (VM), intubação orotraqueal, sondagem vesical e enteral, punções venosas e arteriais). Esse ambiente é desconfortável devido ao uso de equipamentos de monitorização intensiva e ruídos ambientais ${ }^{(1)}$.

Frequentemente, os pacientes críticos encontram-se ansiosos, agitados, confusos, com dor, ocasionados pela imobilidade, lesões e/ou feridas, e, portanto, pelo ambiente adverso que circunda as UTIs. Muitas vezes, há necessidade de iniciar analgesia e sedação como forma de diminuir o desconforto. Neste contexto, devem ser consideradas as condutas terapêuticas, farmacológicas e ambientais, a fim de minimizar as respostas ao ambiente e aos estímulos ${ }^{(2,3)}$.

Diante disso, faz-se necessária a identificação precoce e o manejo adequado das possíveis causas subjacentes da agitação (dor, delirium, hipoxemia, hipoglicemia, hipotensão, síndrome da abstinência do álcool e outras drogas) $)^{(4)}$.

Vale destacar que tanto a dor quanto a ansiedade geram situações de intenso estresse agudo no organismo humano; a resposta humoral caracteriza-se por um aumento nos níveis circulantes de catecolaminas, glucagon e cortisol. Os efeitos metabólicos da resposta dos referidos hormônios ao estresse resultam no aumento do consumo de oxigênio, hiperglicemia, hipercatabolismo protéico e lipídico, retenção hídrica e depuração renal de potássio. Por isso, o uso de sedoanalgesia exige o acompanhamento de forma criteriosa e rigorosa na avaliação dos níveis de sedação alcançados visando evitar sedação profunda, promover diminuição do tempo de VM e de internamento e, consequentemente, redução dos custos hospitalares ${ }^{(5)}$.
A sedação atenua a resposta fisiológica ao estresse favorecendo positivamente o prognóstico do paciente. Quando associada à analgesia, é uma terapêutica essencial na prevenção da síndrome do estresse pós-traumático na UTI(1). Diante disso, os sedativos são comumente usados como adjuvantes da terapia de ansiedade e agitação; alguns pacientes necessitam de sedação para manter a sincronia com a $\mathrm{VM}^{(6)}$.

Deve-se destacar ainda que, no contexto da UTI, é habitual a manutenção de pacientes com alto nível de sedação, totalmente desconectados do meio. No entanto, hoje é evidente a diferença na análise da sedação, pois se busca promover conforto ao paciente, porém mantendo-o passível de despertar facilmente. Isso pode ser alcançado quando objetiva-se atingir um efeito sedativo com o mínimo de fármacos ou em associação. No entanto, atingir adequada, mas não excessiva sedação em pacientes críticos é um processo complexo devido, entre outros fatores, ao metabolismo desses fármacos serem imprevisíveis ${ }^{(1,3)}$

Frente a isso, o paciente em terapia sedativa deve ser avaliado diariamente pela equipe multidisciplinar para que o tratamento seja benéfico a sua recuperação. $A$ necessidade de monitoração constante dos parâmetros de dor, sedação e agitação reflete a natureza dinâmica dos pacientes críticos. Além disso, a reavaliação frequente promove um acompanhamento rigoroso da resposta ao tratamento, favorecendo no manejo de sinais e sintomas indesejáveis e evitando a sedação excessiva ${ }^{(3)}$. Nesse âmbito, destaca-se a atuação da equipe de enfermagem ao prestar cuidados ao paciente sedado e o uso de protocolos de sedação estabelecidos, que podem diminuir a mortalidade, o tempo de VM e de hospitalização ${ }^{(1,7,8)}$.

A avaliação desses pacientes necessita de medidas objetivas, confiáveis e reprodutíveis para o controle da profundidade da sedação 
e para a eficácia da analgesia. Tanto a sedação excessiva quanto a insuficiente são deletérias, sendo assim para a maioria dos pacientes, as metas de sedação visam amenizar a ansiedade, promover o sono, viabilizar os cuidados de enfermagem e a VM e diminuir o consumo de oxigênio pelo miocárdio ${ }^{(3,9)}$. Portanto, a escala ideal para avaliar os níveis de sedação deve orientar a titulação de terapia e possuir validade e confiabilidade ${ }^{(6,10)}$.

A Escala de Ramsay é a mais utilizada em UTI e como um instrumento unidimensional baseado em critérios clínicos. Caracteriza-se numericamente com pontuações que variam de 1 a 6 e, assim, avalia as respostas do paciente graduadas conforme o nível de sedação(1,6,10).

A escala de agitação-sedação de Richmond (Richmond Agitation-Sedation Scale - RASS) abrange de forma precisa o nível de agitação e ansiedade; isto se configura como vantagem sobre a escala de Ramsay ${ }^{(5)}$. Além disso, é um dos instrumentos de avaliação mais válidos, aplicáveis e confiáveis para mensurar a qualidade e profundidade da sedação em pacientes adultos criticamente enfermos. Também é passível de ser utilizado na prática clínica e em protocolos, com o objetivo de minimizar os impactos negativos da sedação excessiva e agitação(4,10).

Como meta da sedação, espera-se paciente calmo que pode ser facilmente acordado com manutenção do ciclo sono-vigília normal, embora alguns necessitem de sedação profunda para promover sincronia com a $\mathrm{VM}^{(6)}$.

Na prática clínica ainda há um estigma relacionado ao fato de a sedação profunda ser supostamente melhor para o paciente crítico suportar os estímulos inerentes à UTI. É comum associá-la às condições convenientes para a prática de enfermagem; assim não há uma legítima consideração das reais necessidades dos pacientes. Portanto, faz-se necessário manter níveis de sedação adequada, padronizar condu- tas e realizar monitoração neurológica criteriosa visando garantir assistência qualificada, o que justifica a realização deste estudo.

Esta pesquisa objetiva avaliar o processo de sedação em pacientes sob ventilação mecânica invasiva (VMI).

\section{MÉTODO}

Estudo transversal, observacional, realizado entre setembro de 2014 e fevereiro de 2015 na UTI de uma instituição pública de ensino de grande porte, localizada em Goiânia/GO. Nesse período foram admitidos 278 pacientes.

Incluíram-se pacientes com idade maior de 18 anos, admitidos para tratamento clínico e/ou cirúrgico, intubados e sedados há mais de 24 horas e com necessidade de VMI. Foram excluídos os maiores de 18 anos em uso de bloqueadores neuromusculares de forma contínua em bomba infusora, com diagnóstico de esclerose lateral amiotrófica (ELA), Miastenia gravis, Guillain Barré e outras doenças neuromusculares.

A coleta de dados foi feita por meio de observação estruturada e participante, utilizando um instrumento designado como 『Formulário de Monitoramento dos Pacientes Sedados区 após realização prévia do teste piloto. Para efeito de caracterização da amostra estudada foram coletados dados gerais como nome, data de nascimento, sexo, etnia, número do prontuário, data de internação no hospital e na UTI, comorbidades, tipo de admissão, causa da admissão, dados de alta e índices de prognóstico e gravidade. Avaliou-se também com esse instrumento a analgosedação, tipo checklist que incluiu informações diárias de cada paciente sobre o nível de consciência (por meio da escala de RASS $^{(12)}$ e Escala de Coma de Glasgow $\left.{ }^{(13)}\right)$, agentes utilizados, tipo de infusão (contínua ou intermitente) e motivo da sedação. 
Na escala de RASS, o paciente alerta e calmo recebe a pontuação 0 (zero) no escore. Há quatro níveis de agitação (que vão do inquieto ao agressivo) graduados na ordem crescente de um a quatro, e há mais cinco níveis de sedação pontuados de um a cinco negativos, onde, por exemplo, a sedação profunda (quando o paciente movimenta-se ou abre os olhos apenas com estimulação física) equivale a -4 e, se não despertável, o paciente obtém RASS -5 ${ }^{(12)}$.

Os índices de gravidade e prognóstico são ferramentas importantes que possibilitam analisar minuciosamente das condições clínicas dos pacientes gravemente enfermos. Neste âmbito, destacam-se o Acute Physiology and Chronic Health Evaluation II (APACHE II), por exemplo, que classifica os pacientes conforme o desvio de 12 variáveis fisiológicas mensuradas e verifica risco de mortalidade; o Simplified Acute Physiology Score 3 (SAPS 3), que também faz uma estimativa do risco de óbito; e o Sequential Organ Failure Assessment (SOFA), que avalia o risco de disfunção orgânica ${ }^{(13,14,15)}$. Neste estudo, tais índices foram coletados a partir dos prontuários no dia da admissão na UTI (Dia 0).

O estudo foi aprovado pelo Comitê de Ética em Pesquisa do Hospital das Clínicas da Universidade Federal de Goiás, protocolo $\mathrm{n}^{\circ}$. 763.827/2014. Os responsáveis legais pelos pacientes foram esclarecidos sobre a pesquisa e somente após a liberação formal, por meio da assinatura do Termo de Consentimento Livre e Esclarecido (TCLE), preencheram-se os instrumentos de coleta de dados.

A coleta foi digitada no programa Statistical Package for Social Science (SPSS) versão 20 para Windows. Realizou-se análise dos dados mediante frequências absolutas e relativas, bem como de medidas de tendência central (médias e desvios-padrão).

\section{RESULTADOS}

Foram incluídos nesse estudo 30 pacientes, totalizando 565 dias de internamento (correspondentes ao resultado da soma dos dias de internação dos 30 pacientes inclusos no estudo) e 263 dias de uso contínuo de sedativos (resultado da soma do tempo que os 30 pacientes estiveram em uso de sedativos). É característica desta UTI admitir pacientes predominantemente no pós-operatório imediato provenientes do centro cirúrgico e hemodinâmica. A maioria deles permaneceu menos de 24 horas na UTI, o que justifica o número reduzido da amostra e o período de coleta dos dados.

Houve predomínio de homens, com média de idade de 61 anos ( $\sigma \pm 15,4$ anos), em insuficiência respiratória aguda (Tabela 1).

Tabela 1 - Características sócio-demográficas e clínicas dos pacientes internados na UTI no período de setembro de 2014 a março de 2015 ( $n=30)$. Goiânia, 2015.

\begin{tabular}{lc}
\hline \multicolumn{1}{c}{ Características } & $\mathbf{n}(\%)$ \\
\hline Sexo & \\
\hline Masculino & $16(53,3)$ \\
Feminino & $14(46,7)$ \\
\hline Idade (anos) & \\
\hline $20-35$ & $02(6,7)$ \\
$36-50$ & $06(20,0)$ \\
$51-65$ & $09(30,0)$ \\
$>65$ & $13(43,3)$ \\
\hline Tipo de admissão & \\
\hline Clínica & $21(70,0)$ \\
Cirurgia emergência & $05(16,7)$ \\
Cirurgia eletiva & $04(13,3)$ \\
\hline Motivo da internação & \\
\hline Insuficiência Respiratória Aguda & $06(20,0)$ \\
Sepse & $05(16,7)$ \\
Afecções neurológicas & $04(13,3)$ \\
Afecções cardiovasculares & $04(13,3)$ \\
Monitorização pós-operatória & $03(10,0)$ \\
Afecções hepáticas & $02(6,7)$ \\
Vascular & $02(6,7)$ \\
Afecções digestivas & $01(3,3)$ \\
Afecções renais/metabólicas & $01(3,3)$ \\
& \\
\hline Mot & \\
\hline & \\
\hline & \\
\hline
\end{tabular}




\begin{tabular}{lc} 
Afecções hematológicas & $01(3,3)$ \\
Pós-parada cardiorrespiratória & $01(3,3)$ \\
\hline Tempo de internação na UTI (dias) & \\
\hline 2-7 dias & $09(30,0)$ \\
$8-15$ dias & $07(23,3)$ \\
$>15$ dias & $14(46,7)$ \\
\hline Tipo de Alta & \\
\hline Óbito & $19(63,3)$ \\
Transferência & $11(36,7)$ \\
\hline Comorbidades & \\
\hline Sim & $20(66,7)$ \\
Não & $10(33,3)$ \\
Diabetes Mellitus* & $14(42,4)$ \\
Hipertensão Arterial Sistêmica* & $10(30,3)$ \\
Insuficiência Renal Crônica* & $03(9,1)$ \\
Acidente Vascular Encefálico* & $02(6,1)$ \\
Neoplasia* & $02(6,1)$ \\
Insuficiência cardíaca congestiva* & $01(3,0)$ \\
Cirrose Hepática* & $01(3,0)$ \\
\hline
\end{tabular}

${ }^{*} n=33$ Fonte: Elaboração própria, 2015.

O escore SOFA obteve mediana geral de 11 $(\sigma \pm 3,8)$; a mediana do APACHE II foi de 28,5 ( $\sigma$ $\pm 9,4)$ e do SAPS3 foi equivalente a $75(\sigma \pm 14,5)$. Verificou-se que a média do SOFA e a probabilidade de óbito gerada pelos índices prognósticos APACHE II e SAPS3 não tiveram médias significativamente diferentes nos pacientes que evoluíram para óbito em comparação com os que receberam alta da UTI. Porém, a maior média de SAPS 3 entre os pacientes que evoluíram para óbito pode ser justificada, pois o perfil de pacientes desta amostra era formado por maioria de idosos e em estado mais grave (Tabela 2).

Tabela 2 - Relação dos índices prognóstico e mortalidade entre os pacientes que evoluiram para óbito versus pacientes que receberam alta da UTI. Goiânia, 2015.

\begin{tabular}{lcc}
\hline Escores & $\begin{array}{c}\text { Médias no } \\
\text { grupo de } \\
\text { óbito }\end{array}$ & $\begin{array}{c}\text { Médias no } \\
\text { grupo de alta }\end{array}$ \\
\hline SOFA & 10 & 9,7 \\
SAPS3 & 73,7 & 68,8 \\
APACHE II & 27,6 & 26,8 \\
\hline
\end{tabular}

Fonte: Elaboração própria, 2015.
Os fatores relacionados à indicação da sedação são apresentados na Tabela 3.

Tabela 3 - Fatores para indicação* da sedação em 30 pacientes. Goiânia, 2015.

\begin{tabular}{ll}
\hline \multicolumn{1}{c}{ Motivo da sedação } & n $(\%)$ \\
\hline Ventilação Mecânica & $28(80,0)$ \\
Conforto & $03(8,6)$ \\
Prevenir aumento da PIC ${ }^{* *}$ & $02(5,7)$ \\
Agitação & $01(2,8)$ \\
Cuidados Paliativos & $01(2,8)$ \\
\hline
\end{tabular}

${ }^{*}$ Nessa análise, verificou-se que alguns pacientes apresentam mais de um motivo para indicação da sedação $(n=35)$.

${ }^{*} P I C$ - pressão intracraniana

Fonte: Elaboração própria, 2015.

A modalidade de administração por infusão contínua esteve presente em 30 pacientes (100\%), sendo que 19 (63,3\%) deles receberam também sedação intermitente durante algum momento da internação na UTI (Tabela 4).

Tabela 4 - Esquemas de sedação* contínua avaliados em 30 pacientes. Goiânia, 2015.

\begin{tabular}{lc}
\hline \multicolumn{1}{c}{ Fármacos } & $\mathbf{n}(\%)$ \\
\hline Fentanil & $23(56,1)$ \\
Midazolam e Fentanil & $17(41,5)$ \\
Midazolam & $01(2,4)$ \\
\hline
\end{tabular}

* Verificou-se que, alguns pacientes foram submetidos a mais de um esquema de sedação durante essa análise (n=41). Fonte: Elaboração própria, 2015.

A dose média do fentanil foi de $130 \mathrm{mcg} /$ $\mathrm{kg} / \mathrm{dia}$ ( $\sigma \pm 80 \mathrm{mcg} / \mathrm{kg} / \mathrm{dia}$ ), enquanto a dose média do midazolam foi de 4,68 mg/kg/dia ( $\sigma$ $\pm 4,04 \mathrm{mcg} / \mathrm{kg} / \mathrm{dia}$ ) (Tabela 5).

O RASS médio observado foi de -4 ( $\sigma \pm$ 2,02). Entre as 263 avaliações realizadas, foram verificados níveis excessivamente altos de sedação em 162 dias $(61,6 \%)$ pela escala de RASS (RASS -4 ou -5).

Evidenciaram-se níveis inadequadamente baixos de sedação (RASS > 0) em 10 avaliações (3,8\%).Verificaram-se também intercorrências associadas à agitação durante as quais a contenção mecânica foi implementada quatro vezes (Tabela 6). 
Tabela 5 - Esquemas de analgosedação* intermitente avaliados em 30 pacientes. Goiânia, 2015.

\begin{tabular}{lc}
\hline \multicolumn{1}{c}{ Fármacos } & $\mathbf{n}(\%)$ \\
\hline Midazolam & $13(27,1)$ \\
Morfina*** $^{*}$ & $12(25,0)$ \\
Haldol*** $^{* *}$ & $09(18,7)$ \\
Clonazepam & $08(16,7)$ \\
Propofol & $02(4,2)$ \\
Diazepam & $02(4,2)$ \\
Fentanil & $01(2,1)$ \\
Lorazepam & $01(2,1)$ \\
\hline
\end{tabular}

* Nas avaliações, constatou-se que os pacientes receberam esquemas variados de analgosedação $(n=48)$.

**Administrada para conforto e controle da dor (antes do curativo e do banho no leito).

*** Utilizado como terapêutica para agitação e ansiedade. Fonte: Elaboração própria, 2015.

Tabela 6 - Eventos adversos associados à agitação durante o período de sedação $(n=16)$. Goiânia, 2015.

\begin{tabular}{lc}
\hline \multicolumn{1}{c}{ Intercorrências } & $\mathbf{n}(\%)$ \\
\hline Retirada inadvertida de & $07(43,7)$ \\
sonda enteral & \\
Assincronia com a VM & $05(31,2)$ \\
Extubação acidental & $03(18,7)$ \\
Acotovelamento de DVE & $01(6,2)$ \\
\hline
\end{tabular}

*Dreno ventricular externo

Fonte: Elaboração própria, 2015.

A média do número de dias com e sem uso contínuo de sedativo foi de $16,96(\sigma \pm 6,56)$ e 6,86 ( $\sigma \pm 8,3)$ respectivamente, sendo que a Escala de Coma de Glasgow atingiu uma média de $7(\sigma \pm 5,6)$.

\section{DISCUSSÃO}

Estima-se que $42 \%$ a $52 \%$ das admissões em UTI são de pacientes idosos ${ }^{(17)}$. A análise da idade dos participantes do estudo permitiu verificar que os dados observados corroboraram com estudos anteriores e apontaram para uma clientela com maioria de idosos nas UTIs ${ }^{(18,17)}$.
O tempo de internação predominante na amostra foi acima de 15 dias, porém não há consenso na literatura sobre o tempo prolongado de internação na UTI. Isto supostamente justifica-se pelo fato de que a maioria dos estudos foi realizada em unidades com amostra mista, ou seja, clínica e cirúrgica ${ }^{(19)}$.

A sedação é um componente fundamental no cuidado aos pacientes criticamente enfermos e submetidos à $\mathrm{VM}^{(20,19,9)}$. Os sedativos em pacientes intubados geralmente são administrados devido à ansiedade e para facilitar o cuidado da equipe de enfermagem ${ }^{(9)}$. O presente estudo observou como principal indicação para a sedação manter a ventilação artificial.

Os sedativos devem ser administrados de forma intermitente ou conforme necessidade do paciente. A infusão em bolus de certas drogas deve ser feita com cautela, principalmente em pacientes em respiração espontânea, devido à probabilidade de provocar depressão respiratória $^{(6)}$. A sedação intermitente foi utilizada, porém não foram constatadas complicações relacionadas a essa modalidade de infusão.

No Brasil, um estudo transversal identificou que o midazolam e o fentanil eram os agentes sedativos mais utilizados $(97,8 \%$ e $91,5 \%$, respectivamente), com o propofol em terceiro lugar (55\%) ${ }^{(22)}$.

A sedação com benzodiazepínicos pode aumentar o tempo de permanência em UTI e os opióides podem produzir efeitos sedativos, mas não diminuem vigília intensa e não tem efeito de amnésia em caso de procedimentos estressantes. Sem amnésia, a maioria dos pacientes que saem da UTI apresenta sintomas de estresse pós-traumático ${ }^{(4,6)}$. O fentanil, opióide de ação curta, pode ter o efeito imediato de sedação e dar conforto ao paciente ${ }^{(6)}$ e tem sido amplamente utilizado na unidade estudada.

A escala de Ramsay é criticada pela sua falta de discriminação clara e descritores específicos 
para diferenciar entre os vários níveis, porém é amplamente usada na prática clínica ${ }^{(6)}$. Na UTI estudada, é a escala predominante nos registros médicos e de enfermagem. RASS é talvez a escala mais extensivamente estudada; foi validada para capacidade de detectar alterações no estado de sedação em dias consecutivos de cuidados na UTI, bem como delirium ${ }^{(8)}$.

Torna-se imperioso destacar que a implementação da escala de RASS possibilita condutas terapêuticas mais direcionadas para os variados estados de agitação e ansiedade - visto que é mais precisa, possui maior mais validade e confiabilidade para mensurar a qualidade e profundidade da sedação em pacientes adultos criticamente enfermos ${ }^{(4,5)}$, o que justificou sua implementação na avaliação dos pacientes incluídos nesse estudo.

Os níveis adequados de sedação (Ramsay 2 a 4 e RASS 0 a -3) estão associados à alta da UTI, portanto manter níveis mínimos de sedação estão relacionados a resultados clínicos favoráveis ${ }^{(5,4)}$. Porém, observou-se maior tendência para a manutenção de níveis profundos de sedação o que pode relacionar à mortalidade, prolongamento no tempo de internação na UTI, tornando-a dispendiosa.

Recomenda-se que os medicamentos sedativos sejam adaptados e mantidos a um nível leve de sedação em pacientes adultos de UTI, exceto haja contraindicação clínica. Por exemplo, em pacientes com aumento da pressão intracraniana ou pacientes de difícil ventilação faz-se necessária completa sedação (Ramsay 5-6). Além disso, o nível de sedação preconizado pode variar conforme a gravidade da doença ${ }^{(4,3)}$.

A sedação extremamente profunda e analgesia deficiente em procedimentos dolorosos devem ser evitadas. Assim como nesse estudo, há outras evidências de grande proporção de pacientes em sedação profunda(23,22,25,26).
Em uma revisão sistemática, constatou-se que 40 a $60 \%$ das avaliações de sedação realizadas são consideradas profundas e que mais de $20 \%$ dos pacientes tem uma sedação sub-ótima (muito profunda ou muito leve). Os autores sugeriram uma abordagem uniforme em monitorar a profundidade e a qualidade da sedação que irá melhorar o cuidado de saúde ${ }^{(25)}$. O risco de os pacientes desenvolverem alteração psicológica subseqüente à internação na UTI foi maior naqueles pacientes sedados e submetidos a níveis de sedação profunda e/ou prolongada (26). Nessa perspectiva, pode-se considerar que os pacientes incluídos nesse estudo estão suscetíveis a esse risco.

Sob o enfoque histórico, têm sido comum a utilização de benzodiazepínicos (ou seja, midazolam e o lorazepam) para sedação de pacientes na UTI. Entretanto, devem-se utilizar, preferencialmente, os esquemas de sedação que incluem sedativos não benzodiazepínicos (propofol ou dexmedetomidina) a fim de beneficiar os desfechos clínicos em pacientes críticos que estão sob VM ${ }^{(4)}$. No entanto, na prática clínica, os benzodiazepínicos são os sedativos mais usados e têm efeito ansiolítico, hipnótico e amnésico ${ }^{(25)}$, pois verificou-se ainda a preferência pela utilização de benzodiazepínicos como o midazolam, nos esquemas de sedação contínua em associação e, principalmente, na modalidade de infusão intermitente. Percebe-se a divergência entre o que é recomendado nos guidelines e o que é implementado na prática.

Nesse estudo, a média do número de dias com uso contínuo de sedativo foi de 16,96; o que pode ser considerado alto, pois pacientes que fazem uso de opióides ou sedativos por mais uma semana podem desenvolver dependência e a rápida retirada desses agentes pode provocar abstinência $^{(6)}$.

O potencial para a abstinência de opióides, benzodiazepínicos, propofol deve ser consi- 
derado após uso por mais do que sete dias de tratamento contínuo. Os guidelines afirmam ainda que a reavaliação frequente da sedação do paciente quanto à necessidade de infusão pode prevenir o prolongamento dos efeitos da sedação ${ }^{(6)}$. Nesse sentido, destaca-se como fundamental a atuação do enfermeiro na avaliação contínua do nível de consciência do paciente crítico sedado e, torna-se necessário mobilizar esse profissional como participante junto à equipe multiprofissional nas decisões terapêuticas em relação aos esquemas de sedação utilizados.

Quando a sedação é feita sem padronizações e quando é manejada a critério do clínico, os pacientes permanecem mais tempo em VM e na UTI. Além disso, esses pacientes permanecem menos tempo acordados, dificultando uma avaliação neurológica adequada ${ }^{(27)}$. Configura-se como problemática encontrada a inexistência de estratégias e de metas de sedação ${ }^{(25)}$.

Estudos recentes têm sido realizados para melhorar o plano de sedação e analgesia com pacientes submetidos à VM, e os resultados mostram que estratégias simples, como o uso de protocolos institucionais e, a interrupção diária da sedação por infusão contínua, resulta na diminuição do tempo de VM e de internação na UTI ${ }^{(7,8,25,26)}$. Embora existam evidências disponíveis, as melhores práticas de sedação ainda são heterogêneas e implantadas de forma diminuta mundialmente. É imperativo modificar essa notória divergência entre a pesquisa e a prática(28).

\section{CONCLUSÃO}

Esse estudo evidenciou que a sedação fez parte da terapêutica do paciente em ventilação mecânica. A amostra predominante foi de homens e com idade média de 61 anos.

Em relação aos esquemas de sedação, não houve padronização no serviço no que se refere a doses e tipos de medicamentos, foram usados principalmente a infusão contínua de fentanil, midazolam isoladamente e de forma associada fentanil e midazolam.

Os pacientes em esquema de sedação contínua tiveram RASS médio de -4 , o que significa que os mesmos tinham níveis excessivamente altos de sedação em 162 dias, o que pode interferir no desmame ventilatório, aumento do tempo de ventilação mecânica, risco de infecções e delirium.

Percebe-se que há certo distanciamento da equipe multiprofissional no que se refere à sedação. Vale salientar que, os enfermeiros desse serviço utilizam em suas avaliações as escalas de Ramsay para avaliar a sedação e Glasgow para avaliar o nível de consciência.

Na unidade onde foi realizado o estudo, recomenda-se a elaboração de protocolo de sedação ou interrupção que inclua a atuação da equipe multiprofissional a fim de evitar sedação profunda e/ou prolongada que é deletéria. No entanto, é imprescindível a sensibilização da equipe para adesão ao protocolo, pois assim poderão traçar estratégias que viabilizem esse processo para a qualidade da assistência e a diminuição dos custos hospitalares.

Recomenda-se a elaboração de estudos com amostra maior e que incluam associações entre os escores de gravidade e o Nursing Activies Score (NAS) ${ }^{(29)}$.

\section{REFERÊNCIAS}

1. Luna, AA, Sousa WA, Ferraz VM. Avaliação de delirium em pacientes em uso de sedativos. Rev Rede de Cuidados em Saúde. 2011; 5(1): 1- 11.

2. Nasraway SAJ, Jacobi J, Murray MJ, Lumb PD. Sedation, analgesia, and neuromuscular blockade of critically ill adult: Revised clinical practice guidelines for 2002. Critical Care Med. 2002; 30(1): 117-118. 
3. Miranda ML, Bersot CD, Villela NR. Sedação, analgesia e bloqueio neuromuscular na unidade de terapia intensiva. Rev HUPE. 2013; 12(3): 102-109.

4. Barr J, Fraser LG, Puntillo K, Ely WE, Devlin JW, Kress JP, et al. Clinical Practice Guidelines for the Management of Pain, Agitation, and Delirium in Adult Patients in the Intensive Care Unit. Critical Care Med. 2013; 41(1): 263-306.

5. Mendes CL, Vasconcelos LCS, Tavares JS, Fontan SB, Ferreira DC, Diniz LAC, et al. Escalas de Ramsay e Richmond são equivalentes para a avaliação do nível de sedação em pacientes gravemente enfermos. Rev Bras Terap Intensiva. 2008; 20(4): 344-348.

6. Jacobi J, Gilles LF, Douglas BC, Richard RR, Dorrie F, Eric TW, et al. Clinical practice guidelines for the sustained use of sedatives and analgesics in the critically ill adult. Critical Care Med. 2002; 30(1): 119-141.

7. Minhas MA, Velasquez AG, Kaul A, Salinas PD, Celi LA. Effect of Protocolized Sedation on Clinical Outcomes in Mechanically Ventilated Intensive Care Unit Patients: A Systematic Review and Meta-analisys of Randomized Controlled Trials. Mayo Clinic Proceedings. 2015; 90(5): 613-623. [included in the review]

8. Scott B, EckleT. The impact of sedation protocols on outcomes in critical illness. Annals of Tanslational Medicine. 2016; 4(2): 33-36. [included in the review]

9. Kress JP, Hall JB. Sedation in the mechanically ventilated patient. Critical Care Med. 2006; 34(10): 2541-2546.

10. Barra DCC, Nascimento RP, Bernardes JFL. Analgesia e sedação em terapia intensiva: recomendações gerais. Rev Min Enferm. 2006; 10(2): 176-180.

11. Yousefi H, Toghyani F, Yazdannik AR, Fazel K. Effect of using Richmond Agitation Sedation Scale on duration of mechanical ventilation, type and dosage of sedation on hospitalized patients in intensive care units. Iranian Journal of Nursing and Midwifery Research. 2015; 20(6): 700-704. [included in the review]

12. Ely EW, Truman B, Shintani A, Thomason JWW, Wheeler AP, Gordon S, et al. Monitoring Sedation Status Over Time in ICU Pacients: Reliability and Validity of Richmond Agitation-Sedation Scale (RASS). Journal of The American Medical Associa- tion (JAMA). 2003; 289(22): 2983-2991. [included in the review]

13. Teasdale G, Jennett B. Assessment of Coma and Impaired Consciousness. Lancet. 1974; 304(7872): 81-84. [included in the review]

14. Knaus WA, Draper EA, Wagner DP, Zimmerman JE. APACHE II: a severity of disease classification system. Crit Care Med. 1985; 13(10): 818-829. [included in the review]

15. Metnitz PG, Moreno RP, Almeida E, Jordan B, Bauer P, Campos RA, et al. - SAPS 3 - From evaluation of the patient to evaluation of the intensive care unit. Part 1:Objectives, methods and cohort description. Intensive Care Med. 2005; 31:13361344. [included in the review]

16. Vincent JL, Moreno R, Takala J, Willatts S, De Mendonça A, Bruining $H$, et al. The SOFA (Sepsis-related Organ Failure Assessment) score to describe organ dysfunction/failure. On behalf of the Working Group on Sepsis-Related Problems of the European Society of Intensive Care Medicine. Intensive Care Med. 1996; 22(7): 707-710. [included in the review]

17. Schein LE, Cesar JA. Perfil de idosos admitidos em unidades de terapia intensiva gerais em Rio Grande, RS: resultados de um estudo de demanda. Rev Bras Epidemiol. 2010; 13(2): 289-301.

18. Juncal VR, Britto LAN, Camelier AA, Messeder $\mathrm{OHC}$, Farias AMC. Impacto clínico do diagnóstico de sepse à admissão em UTI de um hospital privado em Salvador, Bahia. J Bras Pneumol. 2011; 37(1): 85-92.

19. Oliveira ABF, Dias OM, Mello MM, Araújo S, Dragosavac D, Nucci A, et al. Fatores associados à maior mortalidade e tempo de internação prolongado em uma unidade de terapia intensiva de adultos. Rev Bras Ter Intensiva. 2010; 22(3): 250-256.

20. Strom T, Martinussen T, Toft P. A protocol of no sedation for critically ill patients receiving mechanical ventilation: a randomised trial. Lancet. 2010; 375 (9713): 475-80.

21. Tanaka LMS, Azevedo LCP, Park M, Schettino G, Nassar APJ, Réa-Neto A, et al. Early sedation and clinical outcomes of mechanically ventilated patients: a prospective multicenter cohort study. Critical Care. 2014;18(4):R156.

22. Salluh Jl, Dal-Pizzol F, Mello PV, Friedman G, Silva E, Teles JM, et al. Brazilian Research in Intensive 
Care Network. Delirium recognition and sedation practices in critically ill patients: a survey on the attitudes of 1015 Brazilian critical care physicians. J Crit Care. 2009; 24(4): 556-62.

23. Mehta S, McCullagh I, Burry L. Current sedation practices: lessons learned from international surveys. Anesthesiol Clin. 2011; 29(4): 607-624.

24. Shehabi Y, Chan L, Kadiman S, Alias A, Ismail WN, Tan $M$, et al. Sedation depth and long-termmortality in mechanic ally ventilated critically ill adults: a prospective longitudinal multicentre cohort study. Intensive Care Med. 2013; 39: 910-918.

25. Jackson DL, Proudfoot CW, Cann KF, Walsh TS. The incidence of sub-optimal sedation in the ICU: a systematic review. Critical Care. 2009; 13(6): 1-14.

26. Costa JB, Marcon SS, Macedo CRL, Jorge AC, Duarte PAD. Sedação e memórias de pacientes submetidos à ventilação mecânica em unidade de terapia intensiva. Rev Bras Ter Intensiva. 2014; 26(2): 122-128.

27. Trikha A, Rewari, V. Sedation, Analgesia and Muscle Relaxation in the Intensive Care Unit. Indian Journal of Anaesthesia. 2008; 52(5): 620-631.

28. Shinotsuka CR, Salluh JIF. Percepções e práticas sobre delirium, sedação e analgesia em pacientes críticos: uma revisão narrativa. Rev Bras Ter Intensiva. 2013; 25(2): 155-161.

29. Queijo AF, Padilha KG. Nursing Activities Score (NAS): adaptação transcultural e validação para a língua portuguesa. Revista da Escola de Enfermagem da USP. 2009; 43(esp): 1018-1025. [incluída na revisão]
Todos os autores participaram das fases dessa publicação em uma ou mais etapas a seguir, de acordo com as recomendações do International Committe of Medical Journal Editors (ICMJE, 2013): (a) participação substancial na concepção ou confecção do manuscrito ou da coleta, análise ou interpretação dos dados; (b) elaboração do trabalho ou realização de revisão crítica do conteúdo intelectual; (c) aprovação da versão submetida. Todos os autores declaram para os devidos fins que são de suas responsabilidades o conteúdo relacionado a todos os aspectos do manuscrito submetido ao OBJN. Garantem que as questões relacionadas com a exatidão ou integridade de qualquer parte do artigo foram devidamente investigadas e resolvidas. Eximindo, portanto o OBJN de qualquer participação solidária em eventuais imbróglios sobre a materia em apreço. Todos os autores declaram que não possuem conflito de interesses, seja de ordem financeira ou de relacionamento, que influencie a redação e/ou interpretação dos achados. Essa declaração foi assinada digitalmente por todos os autores conforme recomendação do ICMJE, cujo modelo está disponível em http://www. objnursing.uff.br/normas/DUDE_final_13-06-2013.pdf

Recebido: $15 / 05 / 2015$

Revisado: 28/03/2016

Aprovado: 05/04/2016 